\title{
Achados clínico-patológicos e laboratoriais em um equino com síndrome de Schiff-Sherrington
}

\author{
Clinical-Pathological and Laboratorial Findings in a Horse with Schiff-Sherrington Syndrome \\ Antônio Carlos Lopes Câmara', Gustavo Peixoto Braga', Camila Osse de Souza', Igor Louzada Moreira', \\ André Santos Leonardo², Juliana Targino Silva Almeida e Macêdo ${ }^{2}$, Pedro Miguel Ocampos Pedroso², \\ Tayná Cardim Morais Fino' ${ }^{2}$ Antonio Raphael Teixeira Neto' ${ }^{1}$
}

\begin{abstract}
Background: Schiff-Sherrington syndrome is characterized by extensor hypertonicity of the forelimbs and flaccid hindlimbs as a result from the interruption of the ascending inhibitory effect on motor neurons of forelimbs. The main cause is trauma to the spinal cord in thoracolumbar segment and is considered a common condition in companion animals, but rarely reported in large animals. The present study aimed to report the clinical-pathological and laboratorial findings of a horse with Schiff-Sherington syndrome.

Case: A 9-year-old mare weighing $330 \mathrm{~kg}$ was referred for clinical evaluation after a $36 \mathrm{~h}$ evolution of an acute onset of inability to stand. During physical examination the horse was on lateral recumbency and presented normal mental status, spasticity of the forelimbs, flaccid paralysis of the hind limbs, and superficial sensitivity decreased from the $13^{\text {th }}$ thoracic vertebra, reaching the perianal region and hindlimbs. Hematological and biochemical abnormalities included mild neutrophilic leukocytosis, hypoalbuminemia and increased activity of aspartate aminotransferase. Cerebrospinal fluid (CSF) analysis showed yellowish coloration (xanthochromia), turbidity $(+1)$, increased density, hypoproteinemia and erythrophagocytosis. Microbiological culture of CSF was negative. Necropsy revealed muscle hematomas and complete fracture of the $10^{\text {th }}$ and $11^{\text {th }}$ thoracic vertebrae with spinal cord compression. Cross section of the thoracic spinal cord presented severe hemorrhage and cavitations areas in the gray matter. Microscopically, vacuolization of the white matter (status spongiosus) with numerous axonal spheroids was present. In the gray matter, the presence of moderate amount of neurons with eosinophilic cytoplasm, nucleus and retracted picnotic (necrosis), discrete perineuronal edema, and moderate foci of hemorrhage was observed.

Discussion: The definitive diagnosis of thoracic vertebral fracture with spinal cord compression and myelomalacia was conducted by the association of epidemiological, clinical, laboratory and pathological findings. Schiff-Sherington syndrome can occur due to serious injuries in the thoracolumbar region with spinal cord compression, resulting in lower motor neuron paralysis in the hind limbs (flaccid paralysis) and upper motor neuron paralysis in the forelimbs (spasticity). In horses, the thoracic segment is the most affected in cases of vertebral fractures. CSF analysis presented as a crucial tool for diagnosis, because it allowed the observation of xanthochromia and erythrophagocytosis. And it also allowed the exclusion of other causes of acute spinal cord impairment, such as rabies, viral myeloencephalitis, protozoal mieloencefalopathy and trypanosomiasis by Trypanosoma evansi. CSF analysis is an important ancillary method, associated with the epidemiological and clinical findings, in the diagnosis of central nervous system diseases in horses. In large animals with suspected fracture and spinal cord compression, CSF analysis can help in the definitive diagnosis, especially when performing radiographic examination is impossible.
\end{abstract}

Keywords: forelimbs spasticity, hindlimbs paralysis, horse, spinal cord compression.

Descritores: compressão medula espinhal, espasticidade membros torácicos, equino, paralisia membros pélvicos. 


\section{INTRODUÇÃO}

A síndrome de Schiff-Sherrington é caracterizada por hipertonicidade extensora dos membros torácicos e flacidez dos pélvicos, decorrente da interrupção da influência inibitória ascendente sobre os neurônios motores dos membros torácicos [8,12]. A principal causa é trauma à medular espinhal no segmento toracolombar, sendo considerada condição frequente em animais de companhia [1,2], mas raramente descrita em grandes animais [3]. Apesar de rara em equinos, tal síndrome já foi relatada esporadicamente $[4,8]$. O diagnóstico é realizado através da avaliação neurológica e por radiografias [2]. Entretanto, é reconhecida a dificuldade da execução de exames radiográficos na coluna espinhal de animais de grande porte [8], tornando a análise do líquido cefalorraquidiano (LCR) importante coadjuvante para o diagnóstico definitivo [6]. Assim, o presente trabalho objetivou relatar os achados clínico-patológicos e laboratoriais de um equino com síndrome de Schiff-Sherington.

\section{CASO}

As informações relatadas foram obtidas de um equino mestiço, fêmea, com idade aproximada de nove anos e pesando $330 \mathrm{~kg}$. A alimentação consistia de capim elefante (Pennisetum purpureum) cortado e ração farelada (trigo e milho). A égua era utilizada como animal de tração e não existia histórico de vacinação e vermifugação. $\mathrm{Na}$ anamnese, o proprietário relatou que a égua havia parido há sete dias e era criada em sistema semi-intensivo, sendo solta em piquete com seu potro e com outra égua durante o período do dia. No período noturno era mantida em baia individual junto ao potro. Em determinada manhã, a égua foi encontrada na baia em decúbito lateral sem capacidade de assumir estação, sendo medicada com fenilbutazona $(15 \mathrm{~mL}$, via endovenosa, dose única); sem melhora. Assim, o equino foi encaminhado para o Hospital Veterinário para exame clínico após evolução de 36h.

Ao exame clínico observou-se decúbito lateral permanente, comportamento alerta, apetite presente, escore corporal bom, mucosas ocular e oral congestas, e discreta taquicardia. $\mathrm{O}$ exame neurológico revelou estado mental normal, ausência de disfunção dos nervos cranianos, espasticidade dos membros torácicos, paralisia flácida dos membros pélvicos (Figura 1A), e sensibilidade superficial diminuída a partir da $13^{\mathrm{a}}$ vértebra torácica, alcançando a região perianal e membros pélvicos. O reflexo do panículo apresentava-se diminuído caudal às primeiras vértebras lombares. Não havia edema e sensibilidade dolorosa à palpação em toda a extensão da coluna vertebral.

Amostras sanguíneas foram colhidas através de venipunção jugular em tubo a vácuo com e sem anticoagulante EDTA $10 \%$ para realização do hemograma e dosagens bioquímicas (uréia, creatinina, aspartato aminotransferase [AST], proteínas totais e frações). Coletou-se amostra de LCR no espaço lombossacro para análise de acordo com a metodologia de Schwarz \& Piercy [14]. Alíquota do LCR foi encaminhada para cultura microbiológica. Os achados hematológicos e bioquímicos apresentaram como anormalidades, leve leucocitose com neutrofilia, hipoalbuminemia e aumento de atividade da enzima AST (Tabela 1). A análise do LCR apresentou coloração amarelada (xantocromia), aspecto turvo (+1), aumento da densidade, hipoproteinemia e presença de eritrofagocitose. A cultura microbiológica foi negativa (Tabela 2). Diante dos achados clínicos e laboratoriais, principalmente os resultados da análise do LCR, que corroboravam a suspeita clínica de trauma toracolombar, o equino foi submetido à eutanásia.

Na necropsia observaram-se mucosas ocular e oral congestas, hematomas musculares e fratura completa da $10^{\mathrm{a}}$ e $11^{\mathrm{a}}$ vértebras torácicas com compressão medular (Figura 1B). Na secção transversal da medula

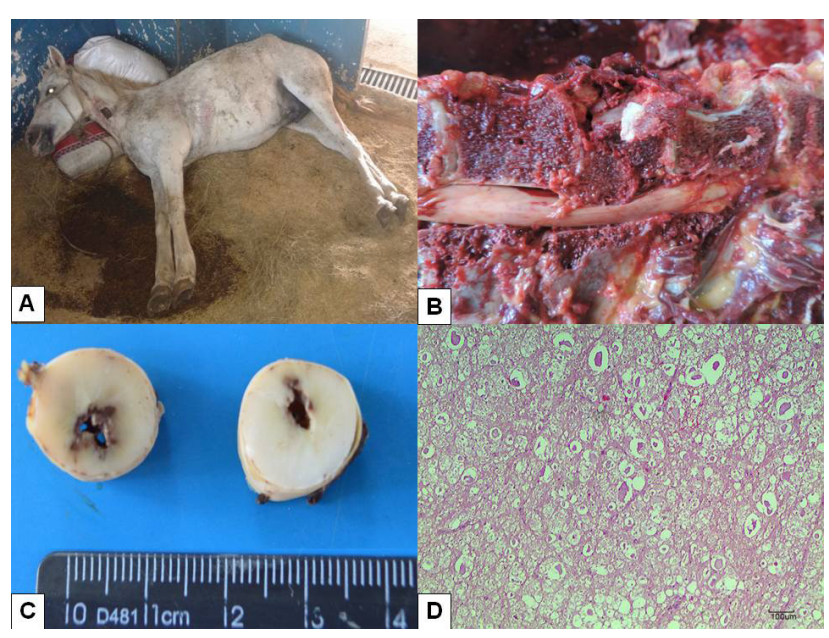

Figura 1. Equino com síndrome de Schiff-Sherington. A- Égua em decúbito lateral com espasticidade dos membros torácicos e paralisia flácida dos membros pélvicos. Observar as marcas no chão da baia decorrentes dos movimentos esporádicos de pedalagem com os membros torácicos. B-Corte longitudinal da coluna vertebral apresentando fratura completa da $10^{\mathrm{a}} \mathrm{e}$ $11^{\mathrm{a}}$ vértebras torácicas com compressão medular. C-Secção transversal da medula torácica demonstrando acentuada hemorragia e áreas de cavitação, principalmente na substância cinzenta. D- Vacuolização da substância branca (status spongiosus) com inúmeros esferoides axonais e substância cinzenta com edema perineuronal, discretos focos de infiltrado neutrófilico e moderados focos de hemorragia. 
Tabela 1. Achados hematológicos e bioquímicos de um equino com síndrome de Schiff-Sherington.

\begin{tabular}{|c|c|c|}
\hline Parâmetro & $\begin{array}{l}\text { Caso } \\
\text { Clínico }\end{array}$ & $\begin{array}{l}\text { Valores de } \\
\text { Referência }{ }^{\text {ef }}\end{array}$ \\
\hline Hematócrito (\%) & 34 & $24-44$ \\
\hline Hemácias $\left(10^{6} / \mu \mathrm{L}\right)$ & 6,77 & $5,5-9,5$ \\
\hline Hemoglobina (g/dL) & 12,3 & $8-14$ \\
\hline $\mathrm{VCM}^{\mathrm{a}}(\mathrm{fL})$ & 50,2 & $39-52$ \\
\hline $\mathrm{CHCM}^{\mathrm{b}}(\%)$ & 34,1 & $31-35$ \\
\hline $\mathrm{PPT}^{\mathrm{c}}(\mathrm{g} / \mathrm{dL})$ & 6,6 & $5,2-7,9$ \\
\hline Albumina (g/dL) & 2 & $2,6-3,7$ \\
\hline Globulina (g/dL) & 4,6 & $2,6-4$ \\
\hline Fibrinogênio (mg/dL) & 200 & $100-400$ \\
\hline Plaquetas & 293.000 & $200.000-500.000$ \\
\hline Leucócitos Totais $(/ \mu \mathrm{L})$ & 13.700 & $6.000-12.000$ \\
\hline Neutrófilos $(/ \mu \mathrm{L})$ & 11.645 & $2.100-9.000$ \\
\hline 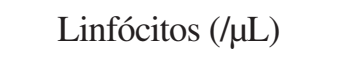 & 2.055 & $900-6.000$ \\
\hline $\mathrm{AST}^{\mathrm{d}}(\mathrm{U} / \mathrm{L})$ & 555 & $226-366$ \\
\hline Creatinina (mg/dL) & 1,8 & $1,2-1,9$ \\
\hline Uréia (mg/dL) & 44 & $21,4-51,3$ \\
\hline
\end{tabular}

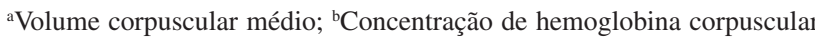

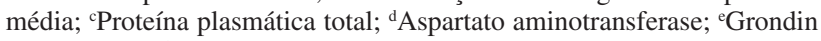

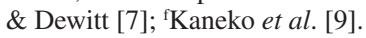

Tabela 2. Resultados da análise e cultura microbiológica do líquido cefalorraquidiano de um equino com síndrome de SchiffSherington.

\begin{tabular}{ccc}
\hline Parâmetro & Caso Clínico & $\begin{array}{c}\text { Valores de } \\
\text { Referência }^{\mathrm{a}}\end{array}$ \\
\hline Aspecto & Turvo $(+)$ & Límpido \\
Cor & Amarelada & Incolor \\
Densidade & 1.010 & $1.004-1.008$ \\
Proteína total $(\mathrm{mg} / \mathrm{dL})$ & 32,8 & $45-61$ \\
Hemácias $(/ \mu \mathrm{L})$ & 1.642 & Raras \\
Leucócitos $(/ \mu \mathrm{L})$ & 5 & $0-7$ \\
Eritrofagocitose & Presente & Ausente \\
Cultura microbiológica & Ausente & Ausente \\
\hline
\end{tabular}

${ }^{\mathrm{a}}$ Furr [6].

torácica havia acentuada hemorragia com áreas de cavitação principalmente na substância cinzenta (Figura 1C). Foram coletados encéfalo, medula espinhal completa e fragmentos de órgãos da cavidade abdominal e torácica. Os fragmentos foram fixados em solução de formol $10 \%$, clivados, processados rotineiramente para histologia e corados pela hematoxilina e eosina (HE).
Os achados microscópicos em fragmento na altura da intumescência torácica incluíram acentuada vacuolização da substância branca (status spongiosus) com inúmeros esferoides axonais. Na substância cinzenta havia moderada quantidade de neurônios com citoplasma eosinofílico, núcleo retraído e picnótico (necrose), edema perineuronal, discretos focos de infiltrado neutrofílico e moderados focos de hemorragia. Células grânulo-adiposas (Gitter) foram raramente observadas em todo fragmento analisado. Em secções da medula cervical e lombar foram observadas apenas discretos focos de hemorragia na substância cinzenta. Tais achados são característicos de mielomalácia (Figura 1D). Os demais órgãos analisados não apresentaram alterações. Fragmentos do sistema nervoso central foram encaminhados para realização de imunofluorescência direta para raiva, com resultado negativo.

\section{DISCUSSÃO}

O diagnóstico definitivo de fratura vertebral torácica com compressão medular e mielomalácia foi realizado através da associação entre os dados epidemiológicos, achados clínicos, laboratoriais e patológicos. A síndrome de Schiff-Sherington pode ocorrer decorrente de lesões graves na região toracolombar com compressão medular, que resultam em paralisia de neurônio motor inferior nos membros pélvicos (paralisia flácida) e paralisia de neurônio motor superior em membros torácicos (espasticidade) [2,4,10,12]. Em equinos, o segmento torácico é o mais afetado em casos de fraturas vertebrais [3].

As alterações laboratoriais, como discreta leucocitose por neutrofilia e aumento da concentração sérica da enzima AST podem ser correlacionados à liberação de cortisol endógeno por estresse e dano muscular devido ao decúbito prolongado, respectivamente [7]. A coleta de LCR no espaço lombossacro de animais suspeitos de trauma medular, mas sem sinais encefálicos, é considerada segura [10]. A densidade acima dos parâmetros fisiológicos é considerada achado inespecífico, pois pode ser influenciado pela técnica de coleta, tipo de anestesia e pressão sanguínea [14]. A coloração amarelada (xantocromia) é indicativo da presença de bilirrubina plasmática, e observada poucas horas após hemorragia subaracnóide, como também os achados de presença de eritrócitos e eritrofagocitose, e pode persistir por duas a quatro semanas $[6,10]$. Este achado é condizente com o histórico de provável ori- 
gem traumática, com início súbito dos sinais clínicos, e achados macroscópicos de necropsia. Outro aspecto importante é que a contaminação por sangue durante a coleta pode influenciar a quantidade de células nucleadas e a concentração de proteína no LCR, quando a contagem de hemácias é superior a 2.000 hemácias/ $\mu \mathrm{L}$ [6]; demonstrando que nossos resultados são fidedignos. Salienta-se que os achados característicos de hemorragia subaracnóide nem sempre são presentes na análise do LCR, que, em muitos casos de trauma medular, pode apresentar-se normal [10].

A análise do LCR apresentou-se crucial para o diagnóstico, pois permitiu a observação de xantocromia e eritrofagocitose, além de propiciar alíquotas para testes microbiológicos e sorológicos. A maior importância foi permitir a exclusão de outras causas agudas de comprometimento medular, como raiva, mieloencefalite viral, mieloencefalopatia protozoária e tripanossomíase por Trypanosoma evansi $[8,10]$. A raiva paralítica usualmente caracteriza-se por piora clínica gradativa, evoluindo para sinais neurológicos encefálicos. Os achados histológicos incluem, na maioria dos casos, meningoencefalite e meningomielite não supurativa com infiltrado perivascular linfoplasmocitário, microgliose focal, neuronofagia e inclusões intracitoplasmáticas (corpúsculos de Negri) [11]. Casos de mieloencefalite viral usualmente apresentam sinais neurológicos de origem cerebral e do tronco encefálico, alterações no LCR (pleocitose neutrofílica, hiperproteinemia e xantocromia) [10] e achados microscópicos bem definidos (encefalomie- lite linfocítica difusa com morte neuronal, satelitose e neuronofagia) [15]. Distúrbios locomotores causados pela infecção por T. evansi podem ser confundidos com fraturas lombares e caracterizam-se por relutância em se mover, ataxia, fraqueza, paresia e incoordenação dos membros pélvicos [8]. Entretanto, a presença de formas flageladas do protozoário nas amostras hematológicas ou patológicas é achado frequente da enfermidade [13]. Equinos com mieloencefalopatia protozoária podem apresentar sintomatologia variada dependendo do local da medula acometido [5]. Resultados da análise do LCR apresentam-se usualmente normais, mas podem revelar discreta pleocitose mononuclear e xantocromia variável [10]. As lesões agudas consistem em áreas multifocais de hemorragias, enquanto as lesões subagudas e crônicas mostram áreas de descoloração que variam de pálido a áreas escurecidas. Microscopicamente, a lesão predominante são áreas de hemorragia multifocal a coalescente, inflamação não supurativa e focos de necrose [5].

A análise do LCR apresenta-se como ferramenta útil, associada aos dados epidemiológicos e sinais clínicos, no diagnóstico de doenças do sistema nervoso central em equinos $[6,10,14]$. Em animais de grande porte com suspeita de fratura com compressão medular, a análise do LCR pode ajudar no diagnóstico definitivo, principalmente quando a realização de exames radiográficos é impossível.

Declaration of interest. The authors report no conflicts of interest. The authors alone are responsible for the content and writing of the paper.

\section{REFERENCES}

1 Albernaz V.G.P, Fabris I.A., Paiva B.R., Coris J.G.F. \& Quitzan J.G. 2016. Traumatic spinal cord injury with SchiffSherrington posture and spontaneous resolution in a dog: MRI evaluation. Acta Veterinaria Brasilica. 10(2): 177-181.

2 Bali M.S., Lang J., Jaggy A., Spreng D., Doherr M.G. \& Forterre F. 2009. Comparative study of vertebral fractures and luxations in dogs and cats. Veterinary and Comparative Orthopaedics and Traumatology. 22(1): 47-53.

3 Borges A.S., Silva D.P.G., Gonçalves S.B., Chiacchio S.B., Amorim R.M., Kuchembuck M.R.G., Vulcano L.C., Bandarra E.P. \& Lopes R.S. 2003. Fraturas vertebrais em grandes animais: estudo retrospectivo de 39 casos (19872002). Arquivo Brasileiro de Medicina Veterinária e Zootecnia. 55(2): 127-132.

4 Chiapetta J.R., Baker J.C. \& Feeney D.A. 1985. Vertebral fracture, extensor hypertonia of thoracic limbs and paralysis of pelvic limbs (Schiff-Sherrington syndrome) in an Arabian foal. Journal of the American Veterinary Medical Association. 186(4): 387-388.

5 Dubey J.P., Lindsay D.S., Saville W.J.A., Reed S.M., Granstrom D.E. \& Speer C.A. 2001. A review of Sarcocystis neurona and equine protozoal myeloencephalitis (EPM). Veterinary Parasitology. 95(2-4): 89-131.

6 Furr M. 2015. Cerebrospinal fluid and the blood-brain barrier. In: Reed S. \& Furr M. (Eds). Equine Neurology. 2nd edn. Ames: Wiley-Blackwell, pp.21-35.

7 Grondin T.E. \& Dewitt S.F. 2010. Normal hematology of the horse and donkey. In: Weiss D.J. \& Wardrop K.J. (Eds). 
A.C.L.Câmara, G.P. Braga, C.O. Souza, et al. 2017. Achados clínico-patológicos e laboratoriais em um equino com síndrome de Schiff-Sherrington. Acta Scientiae Veterinariae.45(Suppl 1): 188.

Schalm's Veterinary Hematology. 6th edn. Ames: Wiley-Blackwell, pp.821-828.

8 Lhamas C.L., Anjos B.L., Pfingstag K.G., Quevedo L.S. \& Duarte C.A. 2015. Síndrome de Schiff-Sherrington em equino - relato de caso. Revista Brasileira de Medicina Veterinária. 37(2): 163-166.

9 Kaneko J.J., Harvey J.W. \& Bruss M.L. 1997. Clinical biochemistry of domestic animals. 5th edn. San Diego: Academic Press, 932p.

10 Mayhew I.G.J. 2009. Physical and chemical causes. In: Mayhew I.G.J. (Ed). Large animal neurology. 2nd edn. Ames: Wiley-Blackwell, pp.294-320.

11 Pedroso P.M.O., Colodel E.M., Gomes D.C., Varaschin M.S., Bezerra Júnior P.S., Barbosa J.D., Tokarnia C.H. \& Driemeier D. 2010. Aspectos clínico-patológicos e imuno-histoquímicos de equídeos infectados pelo vírus da raiva. Pesquisa Veterinária Brasileira. 30(11): 909-914.

12 Riet-Correa F., Riet-Correa G. \& Schild A.L. 2002. Importância do exame clínico para o diagnóstico das enfermidades do sistema nervoso em ruminantes e equídeos. Pesquisa Veterinária Brasileira. 22(4): 161-168.

13 Rodrigues A., Fighera R.A., Souza T.M., Schild A.L., Soares M.P., Milano J. \& Barros C.S.L. 2005. Surtos de tripanossomíase por Trypanosoma evansi no Rio Grande do Sul: aspectos epidemiológicos, clínicos, hematológicos e patológicos. Pesquisa Veterinária Brasileira. 25(4): 239-249.

14 Schwarz B. \& Piercy R.J. 2006. Cerebrospinal fluid collection and its analysis in equine neurological disease. Equine Veterinary Education. 18(5): 243-248.

15 Silva M.L.C.R., Galiza G.J.N, Dantas A.F.M., Oliveira R.O., Iamamoto K., Achkar S.M. \& Riet-Correa F. 2011. Outbreaks of Eastern equine encephalitis in northeastern Brazil. Journal of Veterinary Diagnostic Investigation. 23(3): 570-575. 